\title{
TOTAL PARACOMPACTNESS AND BANACH SPACES
}

\author{
FRANCISCO GALLEGO LUPIAÑEZ
}

(Communicated by Doug W. Curtis)

\begin{abstract}
In this paper, we study some problems related to the Corson theorem. In particular we prove that $c_{0}$ does not fulfil such a theorem; hence this theorem is not valid for all infinite-dimensional Banach spaces. We give also generalizations of Corson's theorem for some infinite-dimensional normed spaces.
\end{abstract}

A topological space is said to be totally paracompact [5] if every open basis contains a locally finite covering. It is known [4] that "every totally paracompact complete metric space is C-scattered" and "every $\sigma$-locally compact paracompact space is totally paracompact". Then, every Banach space is totally paracompact if and only if it is finite dimensional. Thus, every infinite-dimensional Banach space necessarily has an open basis which contains no locally finite covering (i.e. a coarse open basis [3]). We raise the question of how to give an intrinsic description of those infinite-dimensional Banach spaces such that the open basis formed by all open balls is coarse. Corson's theorem is related with this question:

THE CORSON THEOREM [2]. For any covering $\mathscr{U}$ of a reflexive, infinitedimensional Banach space $B$, where $\mathscr{U}$ is formed by bounded, convex sets, there is a point $x$ in $B$ such that each neighborhood of $x$ meets infinitely many elements of $\mathscr{U}$; i.e., $\mathscr{U}$ is not locally finite.

In this paper, we study some problems related to the Corson theorem. In particular, we shall prove that $c_{0}$ does not fulfil such a theorem, hence this theorem is not valid for all infinite-dimensional Banach spaces. We shall give a generalization of the Corson theorem for some infinite-dimensional normed spaces.

THEOREM 1. For every $r>0$, there exists an open covering of $c_{0}$, which is locally finite and is formed by balls of radius $r$.

ProOF. Let $\mathscr{U}=\left\{B_{1}(0)\right\} \cup \mathscr{U}_{+} \cup \mathscr{U}_{-}$, where

$$
\begin{aligned}
\mathscr{U}_{+}=\left\{B_{1}\left(x_{1}, \ldots, x_{i-1}, n+\frac{1}{2}, 0, \ldots\right) \mid i \in \mathbf{N}, n \in \mathbf{N},\right. \\
\left.x_{k} \in\{0\} \cup\left\{p+\frac{1}{2},-q-\frac{1}{2} \mid p, q \in \mathbf{N}\right\}, k \in\{1, \ldots, i-1\}\right\}, \\
\mathscr{U}_{-}=\left\{B_{1}\left(y_{1}, \ldots, y_{j-1},-m-\frac{1}{2}, 0, \ldots\right) \mid j \in \mathbf{N}, m \in \mathbf{N},\right. \\
\left.y_{i} \in\{0\} \cup\left\{p+\frac{1}{2},-q-\frac{1}{2} \mid p, q \in \mathbf{N}\right\}, i \in\{1, \ldots, j-1\}\right\} .
\end{aligned}
$$

Received by the editors June 27, 1986 and, in revised form, February 3, 1987.

1980 Mathematics Subject Classification (1985 Revision). Primary 46A05, 46B05, 54A10, 54D18.

Key words and phrases. Open basis, locally finite covering, Banach spaces, bounded convex sets, normed spaces, total paracompactness.

The results of this paper are contained in the author's Doctoral Thesis, directed by Professor E. Outerelo, to whom the author expresses his hearty thanks for his help in the preparation of this paper. 
We shall prove that

(1) $\mathscr{U}$ covers $c_{0}$.

(2) $\mathscr{U}$ is locally finite in $c_{0}$.

(1) For each $\left(x_{n}\right)_{n \in \mathbf{N}} \in c_{0}$ there is the first natural number $n_{0}$ such that $\left|x_{n}\right|<1$ for every $n \geq n_{0}$.

If $n_{0}=1$, we have $\left|x_{n}\right|<1$ for every $n \geq 1$. Then $\left\|\left(x_{n}\right)_{n \in \mathbf{N}}\right\|_{\infty}<1$ and $\left(x_{n}\right)_{n \in \mathbf{N}} \in B_{1}(0) \in \mathscr{U}$.

If $n_{0}>1$, we have that $\left|x_{n_{0}-1}\right| \geq 1$ and

there is $m=E\left[x_{n_{0}-1}\right] \in \mathbf{N}$ such that $\left|m+\frac{1}{2}-x_{n_{0}-1}\right| \leq \frac{1}{2}<1$ for $x_{n_{0}-1} \geq 1$;

there is $m=E\left[-x_{n_{0}-1}\right] \in \mathrm{N}$ such that $\left|-m-\frac{1}{2}-x_{n_{0}-1}\right| \leq \frac{1}{2}<1$ for $x_{n_{0}-1} \leq-1$. (We write $E[x]$ for the "integral part of $x$ ", the largest integer which does not exceed $x$.)

Let $\left(y_{n}\right)_{n \in \mathbf{N}} \in c_{0}$ be such that $y_{n}=0$ for every $n \geq n_{0}$,

$$
\begin{gathered}
y_{n_{0}-1}= \begin{cases}m+\frac{1}{2} & \text { for } x_{n_{0}-1} \geq 1, \\
-m-\frac{1}{2} \quad \text { for } x_{n_{0}-1} \leq-1, & \text { for each } n<n_{0}-1,\end{cases} \\
y_{n}= \begin{cases}0 & \text { for }\left|x_{n}\right|<1, \\
E\left[x_{n}\right]+\frac{1}{2} & \text { for } x_{n} \geq 1, \\
-E\left[-x_{n}\right]-\frac{1}{2} & \text { for } x_{n} \leq-1 .\end{cases}
\end{gathered}
$$

Then $\left\|\left(x_{n}\right)_{n \in \mathbf{N}}-\left(y_{n}\right)_{n \in \mathbf{N}}\right\|_{\infty}<1$ and $\left(x_{n}\right)_{n \in \mathbf{N}} \in B_{1}\left(\left(y_{n}\right)_{n \in \mathbf{N}}\right) \in \mathscr{U}_{+} \cup \mathscr{U}_{-} \subset \mathscr{U}$.

(2) We shall prove that $\mathscr{U}_{+}$is locally finite in $c_{0}$ (for $\mathscr{U}_{-}$it is analogous) and clearly we shall have that $\mathscr{U}$ is locally finite in $c_{0}$.

If $\mathscr{U}_{+}$is not locally finite in $c_{0}$, there exists $\left(t_{k}\right)_{k \in \mathbf{N}} \in c_{0}$ such that for each $\delta>0$ there is an infinite family $\mathscr{F}_{\delta}$, which is contained in $\mathscr{U}_{+}$such that $B_{\delta}\left(\left(t_{k}\right)_{k \in \mathbf{N}}\right) \cap B \neq$ $\varnothing$ for every $B \in \mathscr{F}_{\delta}$; then the set

$C_{\delta}=\left\{i \in \mathbf{N} \mid\right.$ there is $m \in \mathbf{N}$, there are $x_{k} \in\{0\} \cup\left\{p+\frac{1}{2},-q-\frac{1}{2} \mid p, q \in \mathbf{N}\right\}$

$$
\text { for each } k \in\{1, \ldots, i-1\}
$$

$$
\text { such that } \left.B_{1}\left(x_{1}, \ldots, x_{i-1}, m+\frac{1}{2}, 0, \ldots\right) \in \mathscr{F}_{\delta}\right\}
$$

is nonvoid. Let $0<\delta<\frac{1}{2}$ in the following argument. $C_{\delta}$ can be infinite or finite.

If $C_{\delta}$ is infinite, then $C_{\delta} \supset\left\{i_{n} \mid n \in \mathbf{N}\right\}$ such that $i_{n}<i_{n+1}$ for every $n \in \mathbf{N}$. Then there exist integers such that

$$
\left\{B_{1}\left(x_{1}^{n}, \ldots, x_{i_{n}-1}^{n}, m_{n}+\frac{1}{2}, 0, \ldots\right) \mid n \in \mathbf{N}\right\} \in \mathscr{F}_{\delta}
$$

and for every $n \in \mathbf{N}$, there exists $\left(z_{k}^{n}\right)_{k \in \mathbf{N}} \in c_{0}$ such that

$$
\left(z_{k}^{n}\right)_{k \in \mathbf{N}} \in B_{\delta}\left(\left(t_{k}\right)_{k \in \mathbf{N}}\right) \cap B_{1}\left(x_{1}^{n}, \ldots, x_{i_{n}-1}^{n}, m_{n}+\frac{1}{2}, 0, \ldots\right) .
$$

Thus, from $\left|z_{i_{n}}^{n}-m_{n}-\frac{1}{2}\right|<1$ and $\left|z_{i_{n}}^{n}-t_{i_{n}}\right|<\delta$ for every $n \in \mathbf{N}$ it follows that $m_{n}-\frac{1}{2}<t_{i_{n}}+\delta$ for every $n \in \mathbf{N}$. Since $m_{n} \in \mathbf{N}$ for every $n \in \mathbf{N}$, we have that $t_{i_{n}}>\frac{1}{2}-\delta$ for every $n \in \mathbf{N}$. Then $\left(t_{k}\right)_{k \in \mathbf{N}}$ is not an element of $c_{0}$ and this is a contradiction.

If $C_{\delta}$ is finite, let an $i_{\delta} \in C_{\delta}$. Then

$$
B_{\delta}\left(\left(t_{k}\right)_{k \in \mathbf{N}}\right) \cap B_{1}\left(x_{1}, \ldots, x_{i_{\delta-1}}, m+\frac{1}{2}, 0, \ldots\right) \neq \varnothing
$$

for infinites $\left(x_{1}, \ldots, x_{i_{\delta}-1}, m+\frac{1}{2}\right)$, where $x_{k} \in\{0\} \cup\left\{p+\frac{1}{2},-q-\frac{1}{2} \mid p, q \in \mathbf{N}\right\}$ for each $k \in\left\{1, \ldots, i_{\delta}-1\right\}$ and $m \in \mathbf{N}$. Then, there exists an infinite family $\mathscr{G}_{\delta} \subset \mathscr{F}_{\delta}$ 
formed by balls $B_{1}\left(x_{1}^{n}, \ldots, x_{i_{-}-1}^{n}, m_{n}+\frac{1}{2}, 0, \ldots\right)$ which, we suppose, are pairwise different.

Now, for $i_{\delta}$ let $U\left(2, i_{\delta}-1\right)$ be the set of all $\left(i_{\delta}-1\right)$-samples with repetitions of the elements 0 and 1 .

For each $s \in U\left(2, i_{\delta}-1\right)$, let $c_{s}=\left\{k \in\left\{1, \ldots, i_{\delta}-1\right\} \mid s(k) \neq 0\right\}$.

Then

$$
\begin{gathered}
\left\{B_{1}\left(x_{1}, \ldots, x_{i_{\delta}-1}, m+\frac{1}{2}, 0, \ldots\right) \mid m \in \mathbf{N}, x_{k} \in\{0\} \cup\left\{p+\frac{1}{2},-q-\frac{1}{2} \mid p, q \in \mathbf{N}\right\}\right. \\
\left.\quad \text { for each } k \in\left\{1, \ldots, i_{\delta}-1\right\}\right\} \\
=\bigcup_{s \in U\left(2, i_{\delta}-1\right)}\left\{B_{1}\left(x_{1}, \ldots, x_{i_{\delta}-1}, m+\frac{1}{2}, 0, \ldots\right) \mid m \in \mathbf{N},\right. \\
x_{k} \in \begin{array}{r}
\left\{p+\frac{1}{2},-q-\frac{1}{2} \mid p, q \in \mathbf{N}\right\} \\
\text { for each } \left.k \in c_{s} \text { and } x_{k}=0 \text { for } k \notin c_{s}\right\} .
\end{array}
\end{gathered}
$$

Since $U\left(2, i_{\delta}-1\right)$ is finite and $\mathscr{G}_{\delta}$ is infinite, there exists $s_{0} \in U\left(2, i_{\delta}-1\right)$ and an infinite family $\mathscr{A}_{\delta} \subset \mathscr{G}_{\delta}$ such that

$$
\begin{aligned}
& \mathscr{A}_{\delta}=\left\{B_{1}\left(x_{1}^{n}, \ldots, x_{i_{\delta}-1}^{n}, m_{n}+\frac{1}{2}, 0, \ldots\right) \mid n \in \mathrm{N},\right. \\
& x_{k}^{n}=0 \text { iff } s_{0}(k)=0, \\
& \left.x_{k}^{n} \in\left\{p+\frac{1}{2},-q-\frac{1}{2} \mid p, q \in \mathrm{N}\right\} \text { iff } s_{0}(k) \neq 0\right\} .
\end{aligned}
$$

For every $k \in c_{s_{0}}$ let

$$
S_{k}=\left\{x_{k}^{n} \mid \text { some } B_{1}\left(y_{1}, \ldots, y_{i_{\delta}-1}, m+\frac{1}{2}, 0, \ldots\right) \in \mathscr{A}_{\delta} \text { with } y_{k}=x_{k}^{n}\right\}
$$

and let

$$
S_{i_{\delta}}=\left\{m+\frac{1}{2} \mid m \in \mathbf{N} \text { and some } B_{1}\left(y_{1}, \ldots, y_{i_{\delta}-1}, m+\frac{1}{2}, 0, \ldots\right) \in \mathscr{A}_{\delta}\right\} .
$$

Since card $\mathscr{A}_{\delta} \leq\left(\prod_{k \in c_{s_{0}}}\right.$ card $\left.S_{k}\right) \cdot \operatorname{card} S_{i_{\delta}}$ and $\mathscr{A}_{\delta}$ is infinite, there exists $k_{0} \in$ $c_{s_{0}}$ such that $S_{k_{0}}$ is infinite or $S_{i_{\delta}}$ is infinite.

Suppose $S_{i_{\delta}}$ infinite. Then for each $n \in \mathbf{N}$ there is $\left(w_{k}^{n}\right)_{k \in \mathbf{N}} \in c_{0}$ such that

$$
\left(w_{k}^{n}\right)_{k \in \mathbf{N}} \in B_{\delta}\left(\left(t_{k}\right)_{k \in \mathbf{N}}\right) \cap B_{1}\left(x_{1}^{n}, \ldots, x_{i_{\delta}-1}^{n}, m_{n}+\frac{1}{2}, 0, \ldots\right) .
$$

Thus, from $\left|w_{i_{\delta}}^{n}-t_{i_{\delta}}\right|<\delta$ and $\left|m_{n}+\frac{1}{2}-w_{i_{\delta}}^{n}\right|<1$ for every $n \in \mathbf{N}$ it follows that $m_{n}+\frac{1}{2} \in\left(t_{i_{\delta}}-\frac{3}{2}, t_{i_{\delta}}+\frac{3}{2}\right)$ for every $n \in \mathbf{N}$. This contradicts the assumption that $S_{i_{\delta}}$ is infinite. Analogously if $S_{k_{0}}$ is infinite for some $k_{0} \in\left\{1, \ldots, i_{\delta}-1\right\}$.

Whence, from the assumption that $\mathscr{U}_{+}$is not locally finite in $c_{0}$ we have an infinite subfamily $\mathscr{F}_{\delta}$ of $\mathscr{U}_{+}$which allow us to define a nonvoid set $C_{\delta}$ which is neither finite nor infinite.

In the covering $\mathscr{U}$ we can attain the constant radius of the balls to be an arbitrary number $r>0$, because $r B_{1}(a)=B_{r}(r a)$ and the homotheties in a Banach space are homeomorphisms.

PROPOSITION 1. Let $E$ be a normed space, and $F$ be a subspace of $E$. If $E$ has a locally finite covering by bounded, convex sets, then also there is a locally finite covering of $F$ by bounded, convex sets.

ProOF. Let $\mathscr{U}$ be a locally finite covering of $E$ by bounded convex sets. Then $\mathscr{V}=\{U \cap F / U \in \mathscr{U}, U \cap F \neq \varnothing\}$ is also a locally finite covering of $F$ by bounded, convex sets. 
REMARK. From the last proposition, Theorem 1, and the Corson theorem it follows that $c_{0}$ does not have reflexive, infinite-dimensional Banach subspaces. (This is known; see [1, p. 194].)

THEOREM 2. Let $E$ be an infinite-dimensional normed space.

(1) If there exists a topology $T$ on $E$ such that $(E, T)$ is a $T_{2}$ topological vector space and $\bar{B}_{1}(0)$ is compact in $(E, T)$, then there is no covering of $E$, locally finite in the topology defined by the norm, formed by balls.

(2) If there exists a topology $T$ on $E$ such that the closed balls of $E$ are closed in $(E, T)$ and $\bar{B}_{1}(0)$ is compact in $(E, T)$, then there is no covering of $E$, locally finite in the topology defined by the norm, formed by balls.

(3) If there exists a topology $T$ on $E$ such that $(E, T)$ is $T_{2}$ and for each open, bounded, convex subset of $E$ its closure in the topology defined by the norm, is compact in $(E, T)$, then there is no covering of $E$, locally finite in the topology defined by the norm, formed by bounded, convex sets.

(REMARK. (3) generalizes the Corson theorem.)

PROOF. It is analogous to the proof of the Corson theorem [2].

COROLlARY. If an infinite-dimensional Banach space $B$ is conjugate, then there is no locally finite covering of $B$ by balls.

PROOF. It follows from (1) of Theorem 2 and from the Dixmier-Goldberg-Ruston Theorem [6].

REMARK. Theorem 1 contradicts A. Pełczyński's note [9]: "It verifies the result analogous to Corson's theorem for any Banach space in which there is a weakly closed cone, every closed convex and bounded part of which is weakly compact. Such a cone exists in every Banach space in which there is a weakly convergent sequence which is not norm-convergent." Nevertheless, this idea allows us to give a new generalization of the Corson theorem.

THEOREM 3. Let $E$ be an infinite-dimensional normed space. If there exists a topology $T$ on $E$ such that $(E, T)$ is $T_{2}$ and there is a cone $K$ in $E$, with nonvoid interior in $E$ and such that for every open bounded convex subset of $E$ its closure in the topology defined by the norm on $E$, relativized to $K$, is compact in $(E, T)$, then there is no covering of $E$, locally finite in the topology defined by the norm, formed by bounded, convex sets.

PROOF. The result follows by an argument analogous to the proof of the Corson theorem.

OPEN PROBLEMS.

Problem 1. Give a characterization of those infinite-dimensional normed spaces such that the open basis formed by all open balls is coarse.

Problem 2. Give an intrinsic description of those infinite-dimensional normed spaces $E$ which have a topology $T$ such that $(E, T)$ is a $T_{2}$ topological vector space and $\bar{B}_{1}(0)$ is compact in $(E, T)$.

Problem 3. Give a characterization of those infinite-dimensional normed spaces such that the open basis formed by all open, bounded, convex subsets is coarse.

Problem 4. Does the Banach space $c_{0}(A)$, for an arbitrary index set $A$, admit a locally finite covering by open balls? 
Problem 5. Does the James Hagler space [J. Hagler, A counterexample to several questions about Banach spaces, Studia Math. 60 (1977), 289-308] admit a locally finite covering by open balls?

(Problems 4 and 5 were raised by the referee.)

We thank Professor F. Bombal for his advice about this paper.

\section{REFERENCES}

1. S. Banach, Théorie des opérations linéaires, 2nd ed., Chelsea, New York, 1978.

2. H. H. Corson, Collections of convex sets which cover a Banach space, Fund. Math. 49 (1961), 143-145.

3. H. H. Corson, T. J. McMinn, E. A. Michael, and J. I. Nagata, Bases and local finiteness, Notices Amer. Math. Soc. 6 (1959), 814.

4. D. W. Curtis, Total and absolute paracompactness, Fund. Math. 77 (1973), 277-293.

5. R. M. Ford, Basis properties in dimension theory, Doctoral Dissertation, Auburn Univ., 1963.

6. R. B. Holmes, Geometrical functional analysis and its applications, Springer-Verlag, New York, 1975.

7. J. Horwáth, Topological vector spaces and distributions. I, Addison-Wesley, Reading, Mass., 1966.

8. W. Hurewicz and H. Wallman, Dimension theory, 5th ed., Princeton Univ. Press, Princeton, N.J., 1941.

9. A. Pełczyński, MR 23 \# A2732.

10. H. Toruńczyk, Smooth partitions of unity on some nonseparable Banach spaces, Studia Math. 46 (1973), 43-51.

Departamento de Geometria y Topologia, Facultad de Matematicas, UniVERSIDAD COMPLUTENSE DE MADRID, 28040 MADRID, SPAIN 